\title{
STUDY OF A BROWNIAN IMPULSE
}

\author{
By SopHIE MÉzIÈRES AND BERNARD ROYNETTE \\ Université de Nancy I
}

\begin{abstract}
This paper is concerned with the simulation of a 2-dimensional stochastic differential equation motivated by some physical phenomena of fluid mechanics. The drift and diffusion coefficients of the equation admitting local singularities, we are led to study a particular term of strong perturbation denoted by "Brownian impulse." Our suggestion for the simulation is to replace the singularity by a jump on which our study therefore focuses.
\end{abstract}

1. Introduction. The starting point for this paper is a problem arising in the numerical simulation of some special stochastic process.

We are interested in a 2-dimensional differential equation; it is an evolution equation which models a physical phenomenon of fluid mechanics and presents some interest for the engineers of Electricite De France. The drift and diffusion functions are known by a first experimental procedure and it turns out that they can reach very high values in some points; that is, they have local singularities due to rough and strong variations in the neighborhood of some points. The main example we have in mind are functions which, locally, look like a Gaussian density centered at these points and with a very small variance (see Fig. 1).

They are thus Lipschitz continuous with very large Lipschitz constants in the neighborhood of the point.

Numerical simulation of S.D.E. is quite well-known and many discretization schemes have been described. Most of them are extensions of schemes for ordinary equations. Suitable hypotheses on the coefficients (drift and diffusion) of the S.D.E. are required in all the cases for the convergence of the scheme towards the stochastic process which is the solution of the equation. In general we need the Lipschitz continuity and, sometimes, the boundedness of the coefficients. In our situation, classical discretization schemes contain "constants" which blow up and the convergence does not follow.

We suggest in this paper a method to simulate the stochastic process in such a situation.

Received January 1999; revised July 1999.

AMS 1991 subject classifications. 60H10, 65C20, 65U05.

Key words and phrases. Stochastic differential equation, discretization scheme for simulation. 


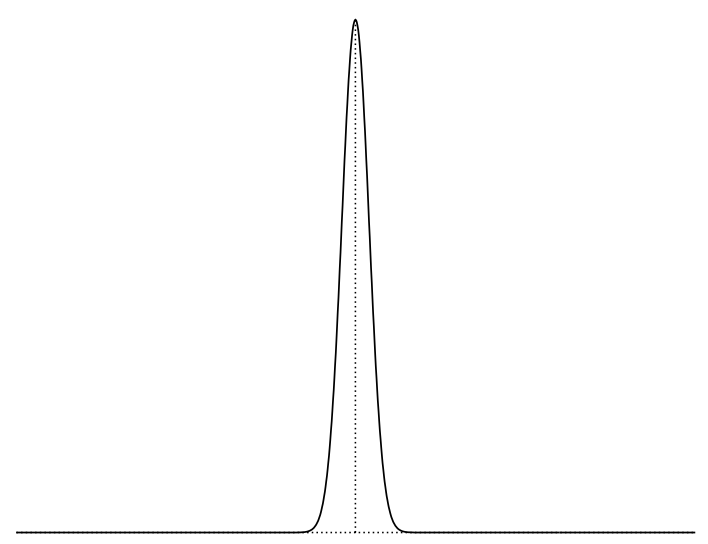

FIG. 1.

Our framework is the following: suppose that the domain is the whole plane $\mathbb{R}^{2}$ and consider the following 2-dimensional S.D.E.:

$\left(E_{1}\right) \quad\left\{\begin{aligned} X_{t}^{1, \varepsilon}= & \int_{0}^{t} \phi\left(\frac{1}{\varepsilon^{2}} X_{s}^{1, \varepsilon}, Y_{s}^{1, \varepsilon}\right) d s \\ Y_{t}^{1, \varepsilon}= & z+\frac{1}{\varepsilon} \int_{0}^{t} \mathbf{1}_{\left[0, \varepsilon^{2}\right]}\left(X_{s}^{1, \varepsilon}\right) d B_{s}+\frac{\delta}{\varepsilon^{2}} \int_{0}^{t} \mathbf{1}_{\left[0, \varepsilon^{2}\right]}\left(X_{s}^{1, \varepsilon}\right) d s \\ & +\int_{0}^{t} \sigma\left(X_{s}^{1, \varepsilon}, Y_{s}^{1, \varepsilon}\right) d \tilde{B}_{s}+\int_{0}^{t} b\left(X_{s}^{1, \varepsilon}, Y_{s}^{1, \varepsilon}\right) d s\end{aligned}\right.$

with $\left(B_{t}\right)_{t \geq 0}$ and $\left(\tilde{B}_{t}\right)_{t \geq 0}$ two independent one-dimensional standard Brownian motions, $z \in \mathbb{R}$, a constant $\delta \in \mathbb{R}$, a little parameter $\varepsilon>0$; and functions $\phi: \mathbb{R}_{+} \times \mathbb{R} \rightarrow \mathbb{R}_{+}^{*}, b: \mathbb{R} \times \mathbb{R} \rightarrow \mathbb{R}$ and $\sigma: \mathbb{R} \times \mathbb{R} \rightarrow \mathbb{R}$ such that the following conditions are satisfied:

(h1) $\phi, b$ and $\sigma$ are Lipschitz continuous (at most linear growth condition is satisfied as a consequence), that is, for the function $\phi$ for instance:

$$
\exists K_{1}>0 ;\left|\phi(z)-\phi\left(z^{\prime}\right)\right| \leq K_{1}\left\|z-z^{\prime}\right\| \quad \forall\left(z, z^{\prime}\right) \in\left(\mathbb{R}_{+} \times \mathbb{R}\right)^{2} .
$$

(h2) $b$ and $\sigma$ are bounded, that is,

$$
\exists K_{2}, K_{3}>0 ;|b(z)| \leq K_{2},|\sigma(z)| \leq K_{3} \quad \forall z \in \mathbb{R}_{+} \times \mathbb{R} .
$$

(h3) $\phi$ is lower bounded, that is,

$$
\exists \eta>0 ; \quad \phi(x, y) \geq \eta>0 \quad \forall(x, y) \in \mathbb{R}_{+} \times \mathbb{R} .
$$

These hypotheses imply the existence and unicity of a strong solution of the equation $\left(E_{1}\right)$.

Let us make two remarks about the equation $\left(E_{1}\right)$.

First we consider only one point of singularity, that is the origin, in common for the drift coefficient and the diffusion one. The situation where there would be several points of singularity is not more complex, we only need to repeat our method for each of these points. 
We have also split up the coefficients of the S.D.E. in two parts: one is singular at the origin, and the other one is regular everywhere. The singularities we have chosen are really simple as being indicator functions. When $\varepsilon$ goes to zero, these two terms blow up rapidly at infinity. In particular the term: $(1 / \varepsilon) \int_{0}^{t} \mathbf{1}_{\left[0, \varepsilon^{2}\right]}\left(X_{s}^{1, \varepsilon}\right) d B_{s}$ increases the oscillations of the stochastic process when $\varepsilon$ decreases to zero. This is the reason why we denote it by "Brownian impulse". We denote by $b$ and $\sigma$ the regular parts of the drift and diffusion coefficients respectively.

In practice such a S.D.E. will be used to approximate the equation in the case where the drift and diffusion coefficients reach very high values at the origin. This modeling requires therefore the definition of a limit value for the coefficients. When a coefficient exceeds this limit value, we suppose it behaves as it is described in equation $\left(E_{1}\right)$.

REMARKS. We index this S.D.E. with the number 1 since we will see later that it is equivalent in law to another S.D.E. that will be indexed with the number 2.

The normalizations in $\varepsilon$ have been chosen in a convenient way to apply scaling properties.

Let us denote by $\left\{Z_{t}^{1, \varepsilon}=\left(X_{t}^{1, \varepsilon}, Y_{t}^{1, \varepsilon}\right)\right\}_{t \geq 0}$ the 2-dimensional stochastic process starting from $Z_{0}^{1, \varepsilon}=(0, z)$ and which is the solution of $\left(E_{1}\right)$. Our motivation is the numerical simulation of the limit process of $Z^{1, \varepsilon}$ as $\varepsilon$ goes to zero. Let us denote it by

$$
Z_{t}^{1}=\lim _{\varepsilon \rightarrow 0} Z_{t}^{1, \varepsilon} \quad \forall t \geq 0 .
$$

As we will see later, a problem appears at the origin. In order to get rid of it, we approximate the process $Z^{1}$ defined in (1) by a classical discretization scheme outside of a little neighborhood of the point $\left(0, Y^{1}\right)$. Let us define the following stopping time:

$$
T^{1, \varepsilon}:=\inf \left\{t \geq 0 ; X_{t}^{1, \varepsilon}=\varepsilon^{2}\right\} .
$$

Most of this work is the determination of the limit law of the random variable $Y_{T_{1, \varepsilon}^{1, \varepsilon}}^{1,}$ when $\varepsilon \rightarrow 0$. Indeed, once we have determined the limit law of $Y_{T^{1, \varepsilon}}^{1, \varepsilon}$, it will be sufficient to approximate $Y^{1}$ with this law when $X^{1}$ reaches the origin. Afterwards we go back normally to the simulation. Hence this paper focuses on the determination of the limit law of $Y_{T^{1, \varepsilon}}^{1, \varepsilon}$ when $\varepsilon$ decreases to zero. This is given by our main result:

THEOREM 1. Let us consider a solution $(s, f, g)$ of the following system (where we denote by $s_{t}$ the derivative of the function $s$ with respect to the time variable $t$, and $s^{\prime}, s^{\prime \prime}$ the first and second derivatives of $s$ with respect to the space variable):

$$
\left\{\begin{array}{l}
\left(s^{\prime} \cdot f\right)_{o} s^{-1}=\frac{1}{\sqrt{\phi}}, \\
\frac{1}{2} f^{2} s^{\prime \prime}+g s^{\prime}+s_{t}=\delta\left(s^{\prime} \cdot f\right)^{2},
\end{array}\right.
$$


where $s: \mathbb{R}_{+} \times \mathbb{R} \rightarrow \mathbb{R}, f: \mathbb{R} \times \mathbb{R} \rightarrow \mathbb{R}, g: \mathbb{R} \times \mathbb{R} \rightarrow \mathbb{R}$ and where, for any fixed $t, s$ is bijective.

Let us next consider the following one-dimensional S.D.E. defined with the preceeding functions $f$ and $g$ :

$$
\left\{\begin{array}{l}
d R_{t}=f\left(t, R_{t}\right) d B_{t}+g\left(t, R_{t}\right) d t, \\
R_{0}=z,
\end{array}\right.
$$

with $\left(B_{t}\right)_{t \geq 0}$ a one-dimensional Brownian motion starting from zero and $z$ a real number. Let us assume that the functions $f$ and $g$ are sufficiently regular to assure this S.D.E. to have a unique solution. Then

$$
\lim _{\varepsilon \rightarrow 0} Y_{T^{1, \varepsilon}}^{1, \varepsilon}=s\left(1, R_{1}\right) \text { in law. }
$$

The plan of the paper is the following: in the second section, we fix our attention on the problem arising in the simulation of $Z^{1}$.

The end of the work is devoted to the proof of Theorem 1. The first step (Section 3) is the transformation of our initial problem into a simpler one on which the required law does not depend any more on the parameter $\varepsilon$. In a first time we prove that in the S.D.E. $\left(E_{1}\right)$, only the coefficients of perturbation (drift and diffusion) play a role when $\varepsilon$ goes to zero. We can then forget the other terms: $\int_{0}^{t} \sigma\left(X_{s}^{1, \varepsilon}, Y_{s}^{1, \varepsilon}\right) d \tilde{B}_{s}$ and $\int_{0}^{t} b\left(X_{s}^{1, \varepsilon}, Y_{s}^{1, \varepsilon}\right) d s$. This leads us to study the law of a new process called $Y^{\varepsilon}$ taken at some new hitting time. In a second time we establish that this process is equivalent in law to another process $Y_{T}$, where $Y$ is a Brownian motion with drift, and $T$ is the hitting time of the level 1 for the process $X$ defined by $X_{t}=\int_{0}^{t} \phi\left(X_{s}, Y_{s}\right) d s$.

The second and last step in the proof of Theorem 1 (Section 4) is concerned with the determination of the law of $Y_{T}$; we can use it to conclude.

Finally, in a last part (Section 5) we illustrate this work with several examples.

2. The simulation problem. To get an idea of what the process $Z^{1, \varepsilon}$ looks like, let us fix $\varepsilon$.

First of all, we remark that the only thing the first component $X^{1, \varepsilon}$ can do is to increase since the function $\phi$ is positive. Then starting from a point of the y-axis, the process $Z^{1, \varepsilon}$ goes towards the right. Its domain of existence is the half plane: $\mathbb{R}_{+} \times \mathbb{R}$.

Inside the strip $\left[0, \varepsilon^{2}\right] \times \mathbb{R}$, the second component $Y^{1, \varepsilon}$ is disturbed by a supplementary Brownian motion with $1 / \varepsilon$ in factor and a supplementary drift with $1 / \varepsilon^{2}$ in factor. Because of the Brownian impulse, the process $Z^{1, \varepsilon}$ oscillates very strongly. We are interested in the law of $Z^{1, \varepsilon}$ when the process reaches the boundary, that is, the line $\left\{x=\varepsilon^{2}\right\}$.

Once the process $Z^{1, \varepsilon}$ leaves the strip $\left[0, \varepsilon^{2}\right] \times \mathbb{R}$, the first component goes on increasing and the second one presents a classical stochastic behavior which is determined by the respective drift and diffusion coefficients $b$ and $\sigma$. Then the process $Z^{1, \varepsilon}$ follows its way towards the right oscillating less strongly 


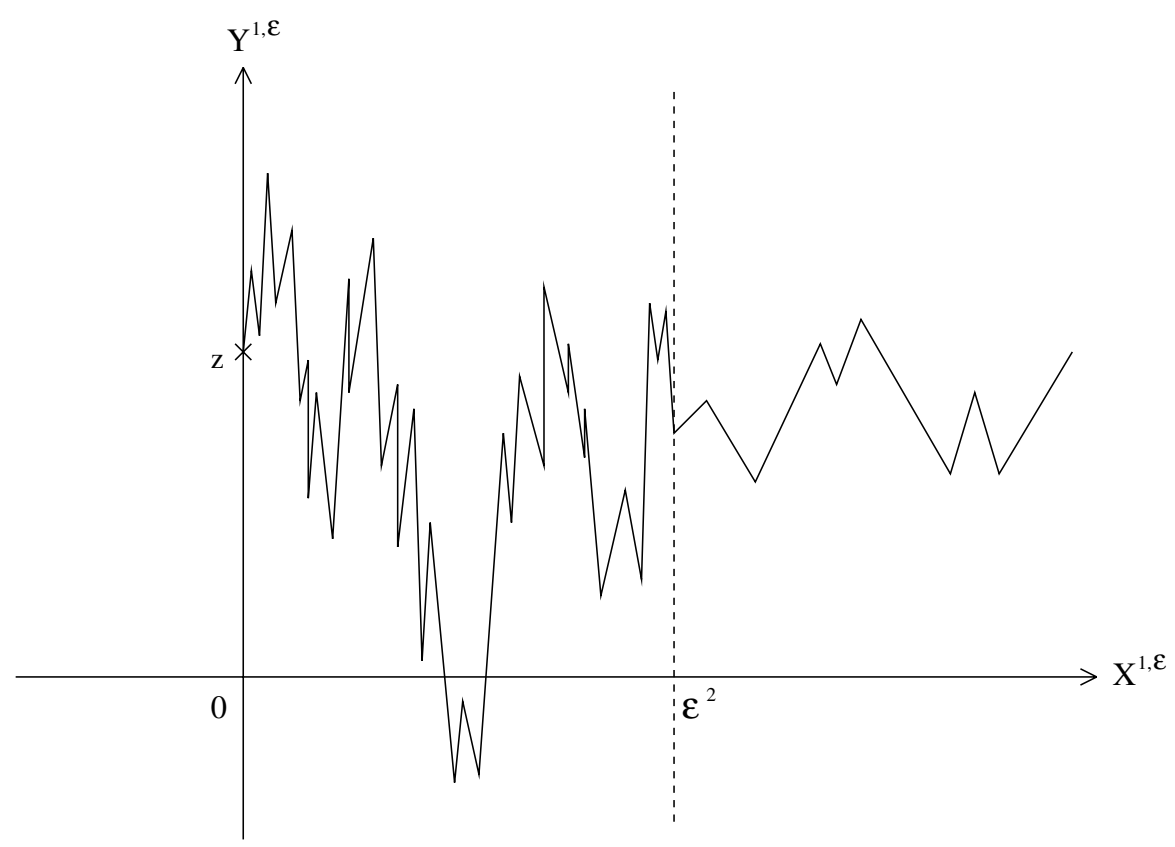

FIG. 2.

than before. Hence, the stopping time $T^{1, \varepsilon}$ is the only exit time of the strip $\left[0, \varepsilon^{2}\right] \times \mathbb{R}$ for this process and it is also the sejourn time of the process in this strip.

$T^{1, \varepsilon}$ is finite. Indeed, since the function $\phi$ is positive and lower bounded, $X^{1, \varepsilon}$ (the first component of $Z^{1, \varepsilon}$ ) starts from the origin and increases. So it will necessarily reach the value $\varepsilon^{2}$ in a finite time.

As we have already said, our motivation is the numerical simulation of the process $Z^{1}$ defined in (1). This is not obvious at first sight. Indeed, whatever the classical discretization scheme we choose, we are not able to approximate $Z_{T^{1, \varepsilon}}^{1, \varepsilon}$ when $\varepsilon \rightarrow 0$.

Let us see this in more details. We use for instance the simplest discretization scheme, the Euler one, and we approximate $Z^{1, \varepsilon}$ in the classical way. In order to simulate the process $Z^{1, \varepsilon}=\left(X^{1, \varepsilon}, Y^{1, \varepsilon}\right)$ in a fixed time interval $[0, T]$, we consider a regular partition of this interval:

$$
0=t_{0}<t_{1}<\cdots<t_{N}=T
$$

with a discretization step we have chosen constant for sake of simplicity:

$$
\Delta t=\frac{T}{N}=t_{k+1}-t_{k} \quad \forall k=0,1, \ldots, N-1
$$


Let us define the discretization scheme $\left\{\bar{Z}_{t_{k}}^{N}=\left(\bar{X}_{t_{k}}^{N}, \bar{Y}_{t_{k}}^{N}\right)\right\}$ by

$$
\begin{aligned}
\bar{X}_{0}^{N}= & X_{0}, \bar{Y}_{0}^{N}=Y_{0} \\
\bar{X}_{t}^{N}= & \bar{X}_{t_{k}}^{N}+\phi\left(\frac{1}{\varepsilon^{2}} \bar{X}_{t_{k}}^{N}, \bar{Y}_{t_{k}}^{N}\right) \cdot\left(t-t_{k}\right) \quad \forall t \in\left[t_{k}, t_{k+1}\right] \\
\bar{Y}_{t}^{N}= & \bar{Y}_{t_{k}}^{N}+\frac{1}{\varepsilon} \mathbf{1}_{\left[0, \varepsilon^{2}\right]}\left(\bar{X}_{t_{k}}^{N}\right) \cdot\left(B_{t}-B_{t_{k}}\right)+\frac{\delta}{\varepsilon^{2}} \mathbf{1}_{\left[0, \varepsilon^{2}\right]}\left(\bar{X}_{t_{k}}^{N}\right) \cdot\left(t-t_{k}\right) \\
& +\sigma\left(\bar{X}_{t_{k}}^{N}, \bar{Y}_{t_{k}}^{N}\right) \cdot\left(\tilde{B}_{t}-\tilde{B}_{t_{k}}\right)+b\left(\bar{X}_{t_{k}}^{N}, \bar{Y}_{t_{k}}^{N}\right) \cdot\left(t-t_{k}\right) \quad \forall t \in\left[t_{k}, t_{k+1}\right]
\end{aligned}
$$

The goal at this point is to establish the convergence of $\left\{\bar{Z}_{t}^{N}\right\}_{t \in[0, T]}$ to $\left\{Z_{t}^{1, \varepsilon}\right\}_{t \in[0, T]}$ when the number of steps $N$ goes to infinity and where $\varepsilon$ is fixed. In order to prove this convergence, for instance in the sense of the a.s. convergence, one usually imposes the following hypotheses on the S.D.E.'s coefficients (which are here supposed to be homogeneous):

- The coefficients are Lipschitz continuous with respect to the space variable.

- The initial data $Z_{0}$ admits a finite moment of even order (i.e., $\exists p \geq$ $\left.1 ; E\left|X_{0}\right|^{2 p}<+\infty, E\left|Y_{0}\right|^{2 p}<+\infty\right)$.

Let us denote the approximation error by

$$
\delta_{t}^{N}:=\left|Y_{t}^{1, \varepsilon}-\bar{Y}_{t}^{N}\right| \text {. }
$$

We commonly prove that there exists $\gamma>0$ such that

$$
E\left(\sup _{t \in[0, T]}\left|\delta_{t}^{N}\right|^{2 p}\right) \leq C(\Delta t)^{\gamma}
$$

in order to deduce that

$$
\sup _{t \in[0, T]} \delta_{t}^{N} \rightarrow 0 \quad \text { p.s. as } N \rightarrow \infty .
$$

We can refer to the Faure's thesis [cf. Faure (1992)] to find a clear and complete review of the first discretization schemes for the S.D.E.: Euler scheme and Milshtein scheme.

Let us now try to approximate the limit process $Z^{1}$. With the hypotheses we have imposed on the S.D.E. $\left(E_{1}\right)$, both conditions above are satisfied in the case where $\varepsilon$ is fixed. When $\varepsilon$ decreases to zero, the coefficients of our S.D.E. $\left(E_{1}\right)$ are no more bounded since the upper bounds explode. We will then obtain an inequality of the form (5) but with a constant $\mathrm{C}$ which will depend of $\varepsilon$ and will blow up at infinity as $\varepsilon$ goes to zero.

From this, it follows that we cannot obtain the convergence of the discretization scheme to the process $Z^{1}$ at the origin. To get rid of this problem, our suggestion is the following: introduce a jump at the origin so that the process will go back from a value $\left(0, \lim _{\varepsilon \rightarrow 0} Y_{T^{1, \varepsilon}}^{1, \varepsilon}\right)$ obtained according to the limit law 
of $Y_{T^{1, \varepsilon}}^{1, \varepsilon}$. Our main interest is now the determination of this law. It is given in Theorem 1. The proof of Theorem 1 is done in two steps.

3. Step 1: Equivalences in law. This part is devoted to the proof of the following fact: the required random variable is equal in law to another random variable the law of which will be determined later on. We proceed in two steps.

3.1. Equivalence in law of $\left(E_{1}\right)$. We will first prove that the process $Z_{T^{1, \varepsilon}}^{1, \varepsilon}$, solution of $\left(E_{1}\right)$ taken at the stopping time $T^{1, \varepsilon}$, has the same limit when $\varepsilon$ decreases to zero, as another process denoted by $Z^{2, \varepsilon}$. This latter is solution of a S.D.E. that turns out to be easier to study than $\left(E_{1}\right)$ and is taken at an appropriate stopping time $T^{2, \varepsilon}$.

Let us consider the 2-dimensional S.D.E.

$$
\left\{\begin{array}{l}
X_{t}^{2, \varepsilon}=\int_{0}^{t} \phi\left(\frac{1}{\varepsilon^{2}} X_{s}^{2, \varepsilon}, Y_{s}^{2, \varepsilon}\right) d s \\
Y_{t}^{2, \varepsilon}=z+\frac{1}{\varepsilon} \int_{0}^{t} \mathbf{1}_{\left[0, \varepsilon^{2}\right]}\left(X_{s}^{2, \varepsilon}\right) d B_{s}+\frac{\delta}{\varepsilon^{2}} \int_{0}^{t} \mathbf{1}_{\left[0, \varepsilon^{2}\right]}\left(X_{s}^{2, \varepsilon}\right) d s
\end{array}\right.
$$

with the same data as in $\left(E_{1}\right)$ : a Brownian motion $\left(B_{t}\right)_{t>0}$, a Lipschitz continuous and lower bounded function $\phi: \mathbb{R}_{+} \times \mathbb{R} \rightarrow \mathbb{R}_{+}^{*}, z, \delta \in \mathbb{R}$, and $\varepsilon>0$. Let us define

$$
T^{2, \varepsilon}:=\inf \left\{t \geq 0 ; X_{t}^{2, \varepsilon}=\varepsilon^{2}\right\} .
$$

and note $\left\{Z_{t}^{2, \varepsilon}=\left(X_{t}^{2, \varepsilon}, Y_{t}^{2, \varepsilon}\right)\right\}_{t \geq 0}$ the solution of $\left(E_{2}\right)$.

We begin with a very useful remark:

REMARK. By definition of $T^{i, \varepsilon}$ (for $i=1,2$ ), we have

$$
\int_{0}^{T^{i, \varepsilon}} \phi\left(\frac{1}{\varepsilon^{2}} X_{s}^{i, \varepsilon}, Y_{s}^{i, \varepsilon}\right) d s=\varepsilon^{2} .
$$

From the fact that the function $\phi$ is lower bounded ((h3)), it follows that

$$
\varepsilon^{2} \geq \int_{0}^{T^{i, \varepsilon}} \eta d s=\eta T^{i, \varepsilon},
$$

that is,

$$
T^{i, \varepsilon} \leq \frac{\varepsilon^{2}}{\eta}, \quad i=1,2
$$

We can therefore establish the following result:

LEMMA 2. Let $\left\{Z_{t}^{1, \varepsilon}=\left(X_{t}^{1, \varepsilon}, Y_{t}^{1, \varepsilon}\right)\right\}_{t \geq 0}$ and $\left\{Z_{t}^{2, \varepsilon}=\left(X_{t}^{2, \varepsilon}, Y_{t}^{2, \varepsilon}\right)\right\}_{t \geq 0}$ be the solution of the S.D.E.'s $\left(E_{1}\right)$ and $\left(E_{2}\right)$ respectively. Then

$$
Y_{T^{1, \varepsilon}}^{1, \varepsilon}-Y_{T^{2, \varepsilon}}^{2, \varepsilon} \rightarrow 0 \quad \text { in } L^{2} \text { and in law, as } \varepsilon \rightarrow 0 .
$$


Proof. In what follows, the quantities $C, C_{i}, i=1,2, \ldots$ will denote constants whose values may change from line to line.

(a) Let us first deal with the first components $X^{1, \varepsilon}$ and $X^{2, \varepsilon}$ of the processes $Z^{1, \varepsilon}$ and $Z^{2, \varepsilon}$ respectively. Using $\left(E_{1}\right)$ and $\left(E_{2}\right)$, we can write

$$
X_{t}^{1, \varepsilon}-X_{t}^{2, \varepsilon}=\int_{0}^{t}\left\{\phi\left(\frac{1}{\varepsilon^{2}} X_{s}^{1, \varepsilon}, Y_{s}^{1, \varepsilon}\right)-\phi\left(\frac{1}{\varepsilon^{2}} X_{s}^{2, \varepsilon}, Y_{s}^{2, \varepsilon}\right)\right\} d s
$$

Then

$$
\begin{aligned}
\sup _{s \leq t}\left|X_{s}^{1, \varepsilon}-X_{s}^{2, \varepsilon}\right| & \leq \int_{0}^{t}\left|\phi\left(\frac{1}{\varepsilon^{2}} X_{s}^{1, \varepsilon}, Y_{s}^{1, \varepsilon}\right)-\phi\left(\frac{1}{\varepsilon^{2}} X_{s}^{2, \varepsilon}, Y_{s}^{2, \varepsilon}\right)\right| d s \\
& \leq K_{1} \int_{0}^{t}\left\{\frac{1}{\varepsilon^{2}}\left|X_{s}^{1, \varepsilon}-X_{s}^{2, \varepsilon}\right|+\left|Y_{s}^{1, \varepsilon}-Y_{s}^{2, \varepsilon}\right|\right\} d s \\
& \quad \text { (since } \phi \text { is Lipschitz continuous) } \\
& \leq K_{1} t \sup _{s \leq t}\left|Y_{s}^{1, \varepsilon}-Y_{s}^{2, \varepsilon}\right|+\frac{K_{1}}{\varepsilon^{2}} \int_{0}^{t}\left(\sup _{u \leq s}\left|X_{u}^{1, \varepsilon}-X_{u}^{2, \varepsilon}\right|\right) d u .
\end{aligned}
$$

From the Gronwall lemma, it follows that

$$
\sup _{s \leq t}\left|X_{s}^{1, \varepsilon}-X_{s}^{2, \varepsilon}\right| \leq K_{1} t \sup _{s \leq t}\left|Y_{s}^{1, \varepsilon}-Y_{s}^{2, \varepsilon}\right| \exp \left(\frac{K_{1} t}{\varepsilon^{2}}\right) .
$$

By applying this inequality with $t=T^{1, \varepsilon} \wedge T^{2, \varepsilon}$ and using (8), we obtain

$$
\sup _{s \leq T^{1, \varepsilon} \wedge T^{2, \varepsilon}}\left|X_{s}^{1, \varepsilon}-X_{s}^{2, \varepsilon}\right| \leq C \varepsilon^{2} \sup _{s \leq T^{1, \varepsilon} \wedge T^{2, \varepsilon}}\left|Y_{s}^{1, \varepsilon}-Y_{s}^{2, \varepsilon}\right| .
$$

In particular if $T^{1, \varepsilon} \wedge T^{2, \varepsilon}=T^{1, \varepsilon}$ (the symetric reasoning - if $T^{1, \varepsilon} \wedge T^{2, \varepsilon}=T^{2, \varepsilon_{-}}$ is similar), we have

$$
\left|X_{T^{1, \varepsilon}}^{2, \varepsilon}-\varepsilon^{2}\right| \leq C \varepsilon^{2} \sup _{s \leq T^{1, \varepsilon} \wedge T^{2, \varepsilon}}\left|Y_{s}^{1, \varepsilon}-Y_{s}^{2, \varepsilon}\right|
$$

Since $\phi$ is lower bounded [cf. (h3)], the time spent to travel from $X_{T^{1, \varepsilon}}^{2, \varepsilon}$ to $X_{T^{2, \varepsilon}}^{2, \varepsilon}=\varepsilon^{2}$ is lower than a constant times the difference $\left|X_{T^{1, \varepsilon}}^{2, \varepsilon}-\varepsilon^{2}\right|$, that is,

$$
\left|T^{1, \varepsilon}-T^{2, \varepsilon}\right| \leq \frac{1}{\eta}\left|X_{T^{1, \varepsilon}}^{2, \varepsilon}-\varepsilon^{2}\right| .
$$

Then

$$
\left|T^{1, \varepsilon}-T^{2, \varepsilon}\right| \leq C \varepsilon^{2} \sup _{s \leq T^{1, \varepsilon} \wedge T^{2, \varepsilon}}\left|Y_{s}^{1, \varepsilon}-Y_{s}^{2, \varepsilon}\right|
$$

This leads us to examine the difference: $\left|Y_{s}^{1, \varepsilon}-Y_{s}^{2, \varepsilon}\right|$. 
(b) Let us now fix our attention to the components $Y^{1, \varepsilon}$ and $Y^{2, \varepsilon}$.

Let us choose the time interval $\left[0, T^{1, \varepsilon} \wedge T^{2, \varepsilon}\right]$ as framework. The two diffusion and drift terms defined on the set $\left\{x \in\left[0, \varepsilon^{2}\right]\right\}$ (the two parts of the singularity in zero) being equal, their difference vanishes. Thus

$$
\begin{aligned}
Y_{s \wedge T^{1, \varepsilon} \wedge T^{2, \varepsilon}}^{1, \varepsilon}-Y_{s \wedge T^{1, \varepsilon} \wedge T^{2, \varepsilon}}^{2, \varepsilon} & \int_{0}^{s \wedge T^{1, \varepsilon} \wedge T^{2, \varepsilon}} \sigma\left(X_{u}^{1, \varepsilon}, Y_{u}^{1, \varepsilon}\right) d \tilde{B}_{u} \\
& +\int_{0}^{s \wedge T^{1, \varepsilon} \wedge T^{2, \varepsilon}} b\left(X_{u}^{1, \varepsilon}, Y_{u}^{1, \varepsilon}\right) d u .
\end{aligned}
$$

We work now in the region $\left\{s \leq T^{1, \varepsilon} \wedge T^{2, \varepsilon}\right\}$ and use the fact that the functions $b$ and $\sigma$ are bounded to obtain

$$
\begin{aligned}
E\left[\sup _{s \leq T^{1, \varepsilon} \wedge T^{2, \varepsilon}}\left|Y_{s}^{1, \varepsilon}-Y_{s}^{2, \varepsilon}\right|^{2}\right] \leq & 2\left\{E\left[\sup _{s \leq T^{1, \varepsilon} \wedge T^{2, \varepsilon}} \int_{0}^{s} \sigma\left(X_{u}^{1, \varepsilon}, Y_{u}^{1, \varepsilon}\right) d \tilde{B}_{u}\right]^{2}\right. \\
& \left.\quad+E\left[\sup _{s \leq T^{1, \varepsilon} \wedge T^{2, \varepsilon}} \int_{0}^{s} b\left(X_{u}^{1, \varepsilon}, Y_{u}^{1, \varepsilon}\right) d u\right]^{2}\right\} \\
\leq & C\left(T^{1, \varepsilon} \wedge T^{2, \varepsilon}\right) \\
\leq & C \varepsilon^{2}
\end{aligned}
$$

On the other hand, we can write

$$
\begin{aligned}
Y_{T^{1, \varepsilon}}^{1, \varepsilon}-Y_{T^{2, \varepsilon}}^{2, \varepsilon}= & \frac{1}{\varepsilon}\left(B_{T^{1, \varepsilon}}-B_{T^{2, \varepsilon}}\right)+\frac{\delta}{\varepsilon^{2}}\left(T^{1, \varepsilon}-T^{2, \varepsilon}\right) \\
& +\int_{0}^{T^{1, \varepsilon}} \sigma\left(X_{s}^{1, \varepsilon}, Y_{s}^{1, \varepsilon}\right) d \tilde{B}_{s}+\int_{0}^{T^{1, \varepsilon}} b\left(X_{s}^{1, \varepsilon}, Y_{s}^{1, \varepsilon}\right) d s .
\end{aligned}
$$

Let us take the square expectation

$$
\begin{gathered}
E\left[\left(Y_{T^{1, \varepsilon}}^{1, \varepsilon}-Y_{T^{2, \varepsilon}}^{2, \varepsilon}\right)^{2}\right] \leq C_{1}\left\{\frac{1}{\varepsilon^{2}} E\left[\left(B_{T^{1, \varepsilon}}-B_{T^{2, \varepsilon}}\right)^{2}\right]+\frac{\delta^{2}}{\varepsilon^{4}} E\left[\left(T^{1, \varepsilon}-T^{2, \varepsilon}\right)^{2}\right]\right. \\
+E\left[\int_{0}^{T^{1, \varepsilon}} \sigma\left(X_{s}^{1, \varepsilon}, Y_{s}^{1, \varepsilon}\right) d \tilde{B}_{s}\right]^{2} \\
\left.+E\left[\int_{0}^{T^{1, \varepsilon}} b\left(X_{s}^{1, \varepsilon}, Y_{s}^{1, \varepsilon}\right) d s\right]^{2}\right\} \\
\leq C_{1}\left\{\frac{1}{\varepsilon^{2}} E\left[\left|T^{1, \varepsilon}-T^{2, \varepsilon}\right|\right]+\frac{\delta^{2}}{\varepsilon^{4}} E\left[\left(T^{1, \varepsilon}-T^{2, \varepsilon}\right)^{2}\right]\right. \\
+E\left[\int_{0}^{T^{1, \varepsilon}} \sigma^{2}\left(X_{s}^{1, \varepsilon}, Y_{s}^{1, \varepsilon}\right) d s\right] \\
\left.+E\left[\int_{0}^{T^{1, \varepsilon}} b\left(X_{s}^{1, \varepsilon}, Y_{s}^{1, \varepsilon}\right) d s\right]^{2}\right\}
\end{gathered}
$$

(cf. the Burkholder-Davis-Gundy inequality) 


$$
\begin{aligned}
\leq C_{1}\{ & \frac{1}{\varepsilon^{2}} E\left(\left|T^{1, \varepsilon}-T^{2, \varepsilon}\right|\right) \\
& \left.+\frac{\delta^{2}}{\varepsilon^{4}} E\left(\left|T^{1, \varepsilon}-T^{2, \varepsilon}\right|^{2}\right)+C_{2} E\left(T^{1, \varepsilon}\right)+C_{2} E\left(T^{1, \varepsilon}\right)^{2}\right\} \\
\leq C_{1}\left\{\frac{1}{\varepsilon^{2}} E\left(\left|T^{1, \varepsilon}-T^{2, \varepsilon}\right|\right)\right. & \quad \text { because of (h2)] } \\
& \left.+\frac{\delta^{2}}{\varepsilon^{4}} E\left(\left|T^{1, \varepsilon}-T^{2, \varepsilon}\right|^{2}\right)+C_{3} \varepsilon^{2}+C_{3} \varepsilon^{4}\right\}[\text { cf. (8)]. }
\end{aligned}
$$

Next, proceeding as in (a), we get

$$
\left(T^{1, \varepsilon}-T^{2, \varepsilon}\right)^{2} \leq\left(X_{s \wedge T^{1, \varepsilon} \wedge T^{2, \varepsilon}}^{1, \varepsilon}-X_{s \wedge T^{1, \varepsilon} \wedge T^{2, \varepsilon}}^{2, \varepsilon}\right)^{2} .
$$

And

$$
\begin{aligned}
\left(X_{t}^{1, \varepsilon}-X_{t}^{2, \varepsilon}\right)^{2}= & {\left[\int_{0}^{t}\left\{\phi\left(\frac{1}{\varepsilon^{2}} X_{s}^{1, \varepsilon}, Y_{s}^{1, \varepsilon}\right)-\phi\left(\frac{1}{\varepsilon^{2}} X_{s}^{2, \varepsilon}, Y_{s}^{2, \varepsilon}\right)\right\} d s\right]^{2} } \\
& \leq\left[K_{1} \int_{0}^{t}\left\{\frac{1}{\varepsilon^{2}}\left|X_{s}^{1, \varepsilon}-X_{s}^{2, \varepsilon}\right|+\left|Y_{s}^{1, \varepsilon}-Y_{s}^{2, \varepsilon}\right|\right\} d s\right]^{2} \\
& \leq\left[K_{1} t \exp \left(\frac{K_{1} t}{\varepsilon^{2}}\right) \sup _{s \leq t}\left|Y_{s}^{1, \varepsilon}-Y_{s}^{2, \varepsilon}\right|\right]^{2} \\
& \leq C_{3} \varepsilon^{4}\left[\sup _{s \leq t}\left|Y_{s}^{1, \varepsilon}-Y_{s}^{2, \varepsilon}\right|\right]^{2}
\end{aligned}
$$

Then

$$
\begin{aligned}
E\left[\left(T^{1, \varepsilon}-T^{2, \varepsilon}\right)^{2}\right] & \leq C_{3} \varepsilon^{4} E\left[\sup _{s \leq t}\left|Y_{s}^{1, \varepsilon}-Y_{s}^{2, \varepsilon}\right|\right]^{2} \\
& \leq C_{3} \varepsilon^{4} E\left[\sup _{s \leq t}\left|Y_{s}^{1, \varepsilon}-Y_{s}^{2, \varepsilon}\right|^{2}\right]
\end{aligned}
$$

We deduce that

(by the Cauchy-Schwarz inequality).

$$
\begin{aligned}
E\left[\left(Y_{T^{1, \varepsilon}}^{1, \varepsilon}-Y_{T^{2, \varepsilon}}^{2, \varepsilon}\right)^{2}\right] \leq & \frac{1}{\varepsilon^{2}} C_{4} \varepsilon^{2} E\left[\sup _{s \leq T^{1, \varepsilon} \wedge T^{2, \varepsilon}}\left|Y_{s}^{1, \varepsilon}-Y_{s}^{2, \varepsilon}\right|^{2}\right]^{\frac{1}{2}} \\
& +\frac{1}{\varepsilon^{4}} C_{4} \varepsilon^{4} E\left[\sup _{s \leq T^{1, \varepsilon} \wedge T^{2, \varepsilon}}\left|Y_{s}^{1, \varepsilon}-Y_{s}^{2, \varepsilon}\right|^{2}\right]+C_{4} \varepsilon^{2}+C_{4} \varepsilon^{4} \\
\leq & \quad C_{4} \sqrt{C_{1}} \varepsilon+C_{4} C_{1} \varepsilon^{2}+C_{4} \varepsilon^{2}+C_{4} \varepsilon^{4} \rightarrow 0 \text { as } \varepsilon \rightarrow 0
\end{aligned}
$$


This proves that $Y_{T^{1, \varepsilon}}^{1, \varepsilon}-Y_{T^{2, \varepsilon}}^{2, \varepsilon}$ tends to zero in $L^{2}$ when $\varepsilon \rightarrow 0$. From this, it follows that $Y_{T^{1, \varepsilon}}^{1, \varepsilon}-Y_{T^{2, \varepsilon}}^{2, \varepsilon}$ converges in law to zero when $\varepsilon \rightarrow 0$. This completes the proof of Lemma 2.

Moreover we have

$$
X_{T^{1, \varepsilon}}^{1, \varepsilon}=X_{T^{2, \varepsilon}}^{2, \varepsilon}=\varepsilon^{2} .
$$

Hence, if $Z_{T^{1, \varepsilon}}^{1, \varepsilon}$ converges in law and the difference $Y_{T^{1, \varepsilon}}^{1, \varepsilon}-Y_{T^{2, \varepsilon}}^{2, \varepsilon}$ converges to zero in $L^{2}$ as $\varepsilon \rightarrow 0$, then $Z_{T^{2, \varepsilon}}^{2, \varepsilon}$ converges in law to the same limit as $Z_{T^{1, \varepsilon}}^{1, \varepsilon}$ when $\varepsilon \rightarrow 0$. This means that in the S.D.E. satisfied by $Y^{1, \varepsilon}$

$$
\begin{aligned}
Y_{t}^{1, \varepsilon}= & z+\frac{\mathbf{1}}{\varepsilon} \int_{0}^{t} \mathbf{1}_{\left[0, \varepsilon^{2}\right]}\left(X_{s}^{1, \varepsilon}\right) d B_{s}+\frac{\delta}{\varepsilon^{2}} \int_{0}^{t} \mathbf{1}_{\left[0, \varepsilon^{2}\right]}\left(X_{s}^{1, \varepsilon}\right) d s \\
& +\int_{0}^{t} \sigma\left(X_{s}^{1, \varepsilon}, Y_{s}^{1, \varepsilon}\right) d \tilde{B}_{s}+\int_{0}^{t} b\left(X_{s}^{1, \varepsilon}, Y_{s}^{1, \varepsilon}\right) d s
\end{aligned}
$$

the only important terms in the strip $\left[0, \varepsilon^{2}\right] \times \mathbb{R}$ are the terms of perturbation (printed in bold-faced type). The last two terms in the right member of this equation play no role when $\varepsilon \rightarrow 0$. Therefore it is sufficient to consider the S.D.E. $\left(E_{2}\right)$ in the following of our work.

A remark will help us to restrict our study. Since we are dealing with the law of our process on the line $\left\{\varepsilon^{2}\right\} \times \mathbb{R}$, it is sufficient to study the process in the strip $\left[0, \varepsilon^{2}\right] \times \mathbb{R}$. We can therefore get rid of the indicator functions in order to keep our notation simple.

It follows from this remark that to determine the limit law of $Y_{T^{1, \varepsilon}}^{1, \varepsilon}$ as $\varepsilon \rightarrow 0$ is equivalent to consider the following 2-dimensional S.D.E.:

$$
\left\{\begin{array}{l}
X_{t}^{\varepsilon}=\int_{0}^{t} \phi\left(\frac{1}{\varepsilon^{2}} X_{s}^{\varepsilon}, Y_{s}^{\varepsilon}\right) d s, \\
Y_{t}^{\varepsilon}=z+\frac{1}{\varepsilon} B_{t}+\frac{\delta}{\varepsilon^{2}} t
\end{array}\right.
$$

in the strip $\left[0, \varepsilon^{2}\right] \times \mathbb{R}$, with the same hypotheses as for $\left(E_{1}\right)$; and determine the limit, when $\varepsilon$ goes to zero, of the law of the process $Y^{\varepsilon}$ taken at the following stopping time:

$$
T^{\varepsilon}:=\inf \left\{t \geq 0 ; X_{t}^{\varepsilon}=\varepsilon^{2}\right\}
$$

3.2. Convergence in law of $Y_{T^{\varepsilon}}^{\varepsilon}$. Let us consider the 2-dimensional S.D.E.:

$$
\left\{\begin{array}{l}
X_{t}=\int_{0}^{t} \phi\left(X_{s}, Y_{s}\right) d s \\
Y_{t}=z+B_{t}+\delta t
\end{array}\right.
$$

where $\left(B_{t}\right)_{t \geq 0}$ is a one-dimensional Brownian motion starting from zero, $z, \delta \in$ $\mathbb{R}$ and $\phi: \mathbb{R}_{+} \times \mathbb{R} \rightarrow \mathbb{R}_{+}^{*}$ are the same data as for the S.D.E. $\left(E_{3}\right)$.

We get a first result: 
LEMMA 3. Let us consider the solution $(X, Y)$ of the equation $\left(E_{4}\right)$, and let us define the pair of processes $\left(\tilde{X}^{\varepsilon}, \tilde{Y}^{\varepsilon}\right)$ by

$$
\left\{\begin{array}{l}
\tilde{X}_{t}^{\varepsilon}:=\varepsilon^{2} X_{\frac{t}{\varepsilon^{2}}}, \\
\tilde{Y}_{t}^{\varepsilon}:=z+B_{\frac{t}{\varepsilon^{2}}}+\frac{\delta}{\varepsilon^{2}} t .
\end{array}\right.
$$

Then $\left(\tilde{X}_{t}^{\varepsilon}, \tilde{Y}_{t}^{\varepsilon}\right)$ is solution in law of $\left(E_{3}\right)$.

The proof of this lemma is a straightforward application of scaling properties, since the pair $\left(\tilde{X}^{\varepsilon}, \tilde{Y}^{\varepsilon}\right)$ has been defined in that aim.

The above lemma tells us that the determination of the limit law of $Y_{T^{\varepsilon}}^{\varepsilon}$ as $\varepsilon \rightarrow 0$ is equivalent to consider the pair $\left(\tilde{X}^{\varepsilon}, \tilde{Y}^{\varepsilon}\right)$ defined by (14) and look for the limit law of $\tilde{Y}^{\varepsilon}$ taken at the following stopping time:

$$
\tilde{T}^{\varepsilon}:=\inf \left\{t \geq 0 ; \tilde{X}_{t}^{\varepsilon}=\varepsilon^{2}\right\}
$$

This means equivalently that: $Y_{T^{\varepsilon}}^{\varepsilon}$ and $\tilde{Y}_{\tilde{T}^{\varepsilon}}^{\varepsilon}$ have the same limit law as $\varepsilon \rightarrow 0$.

Now define

$$
T:=\inf \left\{t \geq 0 ; X_{t}=1\right\},
$$

where $(X, Y)$ is solution of the S.D.E. $\left(E_{4}\right)$.

We can then rewrite $\tilde{T}^{\varepsilon}$ in the form

$$
\begin{aligned}
\tilde{T}^{\varepsilon} & =\inf \left\{t \geq 0 ; \tilde{X}_{t}^{\varepsilon}=\varepsilon^{2}\right\} \\
& =\inf \left\{t \geq 0 ; \varepsilon^{2} X_{\frac{t}{\varepsilon^{2}}}=\varepsilon^{2}\right\} \quad \text { cf. (14) } \\
& =\inf \left\{t \geq 0 ; X_{\frac{t}{\varepsilon^{2}}}=1\right\},
\end{aligned}
$$

It follows that

$$
\tilde{Y}_{\tilde{T}^{\varepsilon}}^{\varepsilon}=z+B_{\frac{T^{\varepsilon}}{\varepsilon^{2}}}+\delta \frac{\tilde{T}^{\varepsilon}}{\varepsilon^{2}}=z+B_{T}+\delta T .
$$

We have then shown that the law of $Y_{T^{\varepsilon}}^{\varepsilon}$ is independent of the parameter $\varepsilon$ since:

$$
Y_{T^{\varepsilon}}^{\varepsilon} \stackrel{(d)}{\simeq} Y_{T}=z+B_{T}+\delta T
$$

4. Step 2: Law of $\boldsymbol{Y}_{\boldsymbol{T}}$. The last step in the proof of Theorem 1 concerns the determination of the law of the process $Y_{T}$, where $(X, Y)$ is a solution of $\left(E_{4}\right)$ and $T$ is the hitting time defined by (16). We are going to associate a one-dimensional process $\left(R_{t}\right)_{t \geq 0}$ to $\left(E_{4}\right)$, and show that some functionals of the pair $\left(t, R_{t}\right)$ are equivalent in law to the components $X$ and $Y$ of the solution to $\left(E_{4}\right)$. Finally the law of $Y_{T}$ will be equal to a simple functional of the process $\left(t, R_{t}\right)$ taken at time 1 . 
Let us recall that we have a Lipschitz continuous and lower bounded function $\phi: \mathbb{R}_{+} \times \mathbb{R} \rightarrow \mathbb{R}_{+}^{*}$ and that we consider the 2-dimensional S.D.E. $\left(E_{4}\right)$ :

$$
\left\{\begin{array}{l}
X_{t}=\int_{0}^{t} \phi\left(X_{s}, Y_{s}\right) d s \\
Y_{t}=z+B_{t}+\delta t
\end{array}\right.
$$

with $\left(B_{t}\right)_{t \geq 0}$ a one-dimensional Brownian motion starting from zero, and $z, \delta \in$ $\mathbb{R}$.

Let us consider an ordinary S.D.E. in which coefficients $f$ and $g$ are assumed to satisfy the usual conditions for the existence and unicity of a solution denoted by $\left(R_{t}\right)_{t \geq 0}$. We assume moreover that this process starts from $z$ at the initial instant. Thus let

$$
R_{t}=z+\int_{0}^{t} f\left(s, R_{s}\right) d B_{s}+\int_{0}^{t} g\left(s, R_{s}\right) d s .
$$

We fix our attention to the pair $\left(t, R_{t}\right)_{t \geq 0}$. The infinitesimal generator associated to this process is

$$
L=\frac{1}{2} f^{2} \frac{\partial^{2}}{\partial x^{2}}+g \frac{\partial}{\partial x}+\frac{\partial}{\partial t} .
$$

Let $s: \mathbb{R}_{+} \times \mathbb{R} \rightarrow \mathbb{R}$ be a function of class $C^{2}$, such that $x \rightarrow s(t, x)$ (where $t$ is fixed) is bijective and $L s=\delta\left(s^{\prime} . f\right)^{2}$.

We apply the Itô Formula to this function $s$ and obtain

$$
\begin{aligned}
s\left(t, R_{t}\right)= & s(0, z)+\int_{0}^{t} \frac{\partial s}{\partial x}\left(u, R_{u}\right) f\left(u, R_{u}\right) d B_{u}+\int_{0}^{t} \frac{\partial s}{\partial x}\left(u, R_{u}\right) g\left(u, R_{u}\right) d u \\
& +\int_{0}^{t} \frac{\partial s}{\partial t}\left(u, R_{u}\right) d u+\frac{1}{2} \int_{0}^{t} \frac{\partial^{2} s}{\partial x^{2}}\left(u, R_{u}\right) f^{2}\left(u, R_{u}\right) d u .
\end{aligned}
$$

Using the properties of the function $s$ :

$$
s\left(t, R_{t}\right)=s(0, z)+\int_{0}^{t} \frac{\partial s}{\partial x}\left(u, R_{u}\right) f\left(u, R_{u}\right) d B_{u}+\delta \int_{0}^{t}\left(\frac{\partial s}{\partial x} \cdot f\right)^{2}\left(u, R_{u}\right) d u .
$$

That is, if we note

$$
Z_{t}:=s\left(t, R_{t}\right)
$$

then

$$
\begin{aligned}
Z_{t} & =s(0, z)+\int_{0}^{t}\left(\frac{\partial s}{\partial x} \cdot f\right)\left(u, R_{u}\right) d B_{u}+\delta \int_{0}^{t}\left(\frac{\partial s}{\partial x} \cdot f\right)^{2}\left(u, R_{u}\right) d u \\
& =Z_{0}+\int_{0}^{t}\left(\frac{\partial s}{\partial x} \cdot f\right)\left(s^{-1}\left(u, Z_{u}\right)\right) d B_{u}+\delta \int_{0}^{t}\left(\frac{\partial s}{\partial x} \cdot f\right)^{2}\left(s^{-1}\left(u, Z_{u}\right)\right) d u \\
& =Z_{0}+\int_{0}^{t} c\left(u, Z_{u}\right) d B_{u}+\delta \int_{0}^{t} c^{2}\left(u, Z_{u}\right) d u \quad \text { with } c:=\left(\frac{\partial s}{\partial x} \cdot f\right)_{\circ} s^{-1}
\end{aligned}
$$


Now define

$$
\begin{aligned}
& A(t)=\int_{0}^{t} c^{2}\left(u, Z_{u}\right) d u, \\
& \tau(t)=A^{-1}(t), \text { that is, } A(\tau(t))=t .
\end{aligned}
$$

Then we have

$$
Z_{t}=Z_{0}+\beta_{A(t)}+\delta A(t),
$$

where $\left(\beta_{t}\right)_{t \geq 0}$ is a one-dimensional Brownian motion [by Dubbins-Schwartz theorem; cf., for instance, Revuz and Yor (1994), Theorem 1.6, page 173]. Hence at time $\tau(t)$, we get

$$
Z_{\tau(t)}=Z_{0}+\beta_{t}+\delta t
$$

On the other hand, $\tau$ is defined by (18). let us derive this equality and assume that $c^{2}>0$ :

$$
\tau^{\prime}(t)=\frac{1}{c^{2}\left(\tau(t), Z_{\tau(t)}\right)}
$$

Then

$$
\begin{aligned}
\tau(t) & =\int_{0}^{t} \frac{d s}{c^{2}\left(\tau(s), Z_{\tau(s)}\right)}=\int_{0}^{t} \frac{d s}{c^{2}\left(\tau(s), Z_{0}+\beta_{s}+\delta s\right)} \quad \text { [cf. (19)] } \\
& =\int_{0}^{t} \phi\left(\tau(s), Z_{0}+\beta_{s}+\delta s\right) d s
\end{aligned}
$$

with the notation

$$
\phi(t, z):=\frac{1}{c^{2}(t, z)} .
$$

Next let us denote

$$
\left\{\begin{array}{l}
X_{t}=\tau(t), \\
Y_{t}=Z_{\tau(t)}=s(0, z)+\beta_{t}+\delta t .
\end{array}\right.
$$

Therefore the pair $\left(X_{t}, Y_{t}\right)_{t \geq 0}$ is a solution of $\left(E_{4}\right)$ :

$$
\left\{\begin{array}{l}
X_{t}=\int_{0}^{t} \phi\left(X_{s}, Y_{s}\right) d s \\
Y_{t}=s(0, z)+\beta_{t}+\delta t
\end{array}\right.
$$

Hence we can rewrite the hitting time of level 1 for $X$ in the form

$$
T=\inf \{t \geq 0 ; \tau(t)=1\} .
$$

Because of (18) and (20), we have

$$
T=A(\tau(T))=A(1) .
$$


It follows that the law of $Y_{T}$ is equal to

$$
Y_{T}=Z_{\tau(T)}=Z_{1} \stackrel{(d)}{\simeq} s\left(1, R_{1}\right)
$$

We have obtained the required law:

$$
\lim _{\varepsilon \rightarrow 0} Y_{T^{1, \varepsilon}}^{1, \varepsilon} \stackrel{(d)}{\simeq} s\left(1, R_{1}\right)
$$

This achieves the proof of Theorem 1 .

REMARK. In practice, the function $\phi$ will be a given data of the problem and we will have to solve the following system in order to determine the functions $c, s, f$ and $g$ :

$$
\left\{\begin{array} { l } 
{ c = \frac { 1 } { \sqrt { \phi } } , } \\
{ c = ( s ^ { \prime } \cdot f ) _ { \circ } s ^ { - 1 } , } \\
{ L s = \delta ( s ^ { \prime } \cdot f ) ^ { 2 } , }
\end{array} \Longleftrightarrow \left\{\begin{array}{l}
\left(s^{\prime} \cdot f\right)_{\circ} s^{-1}=\frac{1}{\sqrt{\phi}}, \\
\frac{1}{2} f^{2} s^{\prime \prime}+g s^{\prime}+s_{t}=\delta\left(s^{\prime} \cdot f\right)^{2},
\end{array}\right.\right.
$$

$\phi$ being the only known data, the problem facing us is to solve a system of two equations with three unknowns. There is therefore a great flexibility in the choice of the solution $(s, f, g)$ to this system.

This result is the main argument for our method of simulation of the process $Z$ since it implies a regular situation in place of a singular one (as described in the introduction). Indeed, we urge the 2-dimensional process $Z^{1, \varepsilon}=\left(X^{1, \varepsilon}, Y^{1, \varepsilon}\right)$ to jump as soon as the component $X^{1, \varepsilon}$ reaches zero. It is therefore sufficient, because of Theorem 1, to simulate the process $R$ which is solution of the S.D.E. (4). If the function $\phi$ is not too bad, this presents no difficulty using classical methods.

5. Some examples. Finally let us collect miscellaneous examples to illustrate our result (Theorem 1).

5.1. Local martingale. We are first going to consider the simple case where the process $\left(R_{t}\right)_{t \geq 0}$ that we are led to simulate in order to get our limit law (by Theorem 1 ) is a local martingale.

Let us work, in the interest of simplicity, with $\delta=0$. And let us consider a convenient function $\phi: \mathbb{R}_{+} \times \mathbb{R} \rightarrow \mathbb{R}_{+}^{*}$, that is $\phi$ satisfies the hypotheses (h1) and (h3). Our purpose is to obtain the limit law $\lim _{\varepsilon \rightarrow 0} Y_{T^{1, \varepsilon}}^{1, \varepsilon}$ where $\left(X^{1, \varepsilon}, Y^{1, \varepsilon}\right)$ is solution of a 2 -dimensional S.D.E. of the type $\left(E_{1}\right)$ with any suitable functions $\sigma$ and $b$ (we have seen that the limit law does not take into account the corresponding terms). By Theorem 1, we have therefore to solve the 2-dimensional system (3) and to determine some functions $f, g$ and $s$. Let us remark that in the case $\delta=0$, the function $s: \mathbb{R}_{+} \times \mathbb{R} \rightarrow \mathbb{R}_{+}^{*}$ is a scale 
function for the defined operator $L$, that is, $L s=0$; and the system that we have to solve becomes

$$
\left\{\begin{array}{l}
\left(s^{\prime} \cdot f\right)_{\circ} s^{-1}=\frac{1}{\sqrt{\phi}}, \\
\frac{1}{2} f^{2} s^{\prime \prime}+g s^{\prime}+s_{t}=0 .
\end{array}\right.
$$

Let us choose here the natural scale for $L$ :

$$
s(t, x)=x
$$

and

$$
\begin{gathered}
f(t, x)=\frac{1}{\sqrt{\phi(t, x)}}, \\
g(t, x) \equiv 0 .
\end{gathered}
$$

Then the process $\left(R_{t}\right)_{t \geq 0}$ defined by (4) is a local martingale as a solution of the following S.D.E.:

$$
d R_{t}=\frac{1}{\sqrt{\phi\left(t, R_{t}\right)}} d B_{t} .
$$

Under the hypotheses required on the function $\phi$, this equation admits a unique solution and we get the limit law by Theorem 1 . This law is given by a local martingale:

$$
\lim _{\varepsilon \rightarrow 0} Y_{T^{1, \varepsilon}}^{1, \varepsilon} \stackrel{(d)}{\simeq} s\left(1, R_{1}\right) \stackrel{(d)}{\simeq} R_{1} \stackrel{(d)}{\simeq} z+\int_{0}^{1} \frac{1}{\sqrt{\phi\left(s, R_{s}\right)}} d B_{s} .
$$

Let us now consider some explicit examples with given functions $\phi$.

5.2. Brownian motion with drift. In the same situation where $\delta=0$ (corresponding to a system (22) and a scale function $s: L s=0$ ), let us now consider the case of a S.D.E. $\left(E_{1}\right)$ determined by suitable functions $b, \sigma$ and

$$
\phi(t, x)=\frac{1}{x^{2}}
$$

It is obvious that this function does not suit to the hypotheses (h1) and (h3). So let us truncate it with as much regularity as possible by defining

$$
\phi_{\eta, M}(t, x):= \begin{cases}\frac{1}{x^{2}}, & \text { if } x \in]-M,-\eta] \cup[\eta, M[, \\ \frac{1}{M^{2}}, & \text { if } x \in]-\infty,-M] \cup[M,+\infty[, \\ \frac{1}{\eta^{2}} & \text { if } x \in]-\eta, \eta[,\end{cases}
$$

with two real positive numbers: a small $\eta$ and a big $M$. 
This truncated function satisfies (h1) and (h3), so we can apply our result to it. We have then to solve the system (22). Let us choose here functions that do not depend on the first variable and $f \equiv 1$, so we get

$$
s_{\eta, M}^{\prime}(x)=\frac{1}{\sqrt{\phi_{\eta, M}\left(s_{\eta, M}(x)\right)}}
$$

and

$$
\frac{1}{2} s_{\eta, M}^{\prime \prime}(x)+g_{\eta, M}(x) s_{\eta, M}^{\prime}(x)=0
$$

Let us first solve (23). $\phi_{\eta, M}$ being lower bounded and Lipschitz continuous, there exists a unique increasing function $s_{\eta, M}$ solution of (23). This one is bijective. In the neighborhood of the points such that $\phi_{\eta, M}(x)=1 / x^{2}$, that is on $]-M,-\eta] \cup[\eta, M[$, the solution of (23) gives us the following scale function:

$$
s_{\eta, M}(x)=e^{x} .
$$

Let us now extend $s_{\eta, M}$ on the whole real axis by defining

$$
s_{\eta, M}(x)= \begin{cases}e^{x}, & \text { if } x \in]-M,-\eta] \cup[\eta, M[ \\ M x+M^{2}+e^{-M}, & \text { if } x \in]-\infty,-M], \\ M x-M^{2}+e^{M}, & \text { if } x \in[M,+\infty[ \\ \frac{\sinh \eta}{\eta} x+\cosh \eta, & \text { if } x \in]-\eta, \eta[\end{cases}
$$

By (24), we obtain

$$
g_{\eta, M}(x)= \begin{cases}-\frac{1}{2}, & \text { if } x \in]-M,-\eta] \cup[\eta, M[ \\ 0, & \text { if not. }\end{cases}
$$

Then we are led (Theorem 1) to consider the S.D.E.

$$
R_{t}^{\eta, M}=z+B_{t}+\int_{0}^{t} g_{\eta, M}\left(R_{s}^{\eta, M}\right) d s
$$

For any fixed $\eta$ and $M$, this equation admits a unique solution $\left(R_{t}^{\eta, M}\right)_{t \geq 0}$.

Let now $\eta \rightarrow 0$ and $M \rightarrow+\infty$. We obviously have

$$
\lim _{\eta \rightarrow 0, M \rightarrow+\infty} g_{\eta, M}(x)=-\frac{1}{2},
$$

and this is not difficult to convince ourselves that the solution $\left(R_{t}^{\eta, M}\right)_{t \geq 0}$ of (25) converges to a unique process $\left(R_{t}\right)_{t \geq 0}$ which is solution of the S.D.E.

$$
R_{t}=z+B_{t}-\frac{t}{2}
$$

This is a Brownian motion with drift starting from $z$. 
From Theorem 1, we get finally for the solution $\left(X^{1, \varepsilon}, Y^{1, \varepsilon}\right)$ of $\left(E_{1}\right)$ with $\phi(t, x)=1 / x^{2}$ and any suitable functions $\sigma$ and $b$

$$
\lim _{\varepsilon \rightarrow 0} Y_{T^{1, \varepsilon}}^{1, \varepsilon} \stackrel{(d)}{\simeq} s\left(1, R_{1}\right) \stackrel{(d)}{\simeq} \exp R_{1} \stackrel{(d)}{\simeq} \exp ^{\left(z-\frac{1}{2}\right)} \exp ^{B_{1}}
$$

The density is therefore given by

$$
p(x)=\frac{1}{\sqrt{2 \pi}} \exp \left(z-\frac{1}{2}\right) \exp \left(x-\frac{x^{2}}{2}\right) .
$$

5.3. Bessel process. Let us now consider the situation where the function $\phi$ has the more general form

$$
\phi(t, x)=\frac{1}{x^{2 \alpha}} \quad \text { with } \alpha \geq 0 .
$$

The same truncation argument as before allows us to apply Theorem 1 to this kind of function and we choose again $\delta=0$.

So we have to solve the system (22) that becomes

$$
\left\{\begin{array}{l}
{\left[\left(s^{\prime} \cdot f\right)_{o} s^{-1}\right](t, x)=x^{\alpha}} \\
\frac{1}{2} f^{2}(t, x) s_{x x}(t, x)+g(t, x) s_{x}(t, x)+s_{t}(t, x)=0 .
\end{array}\right.
$$

Let us choose here

$$
f(t, x) \equiv 1
$$

and a function $g$ of the form:

$$
g(t, x)=\frac{C}{x}
$$

with $C$ a constant to be chosen later.

We obtain therefore

$$
\begin{aligned}
& s^{\prime}{ }_{o} s^{-1}(t, x)=x^{\alpha} \\
& \frac{1}{2} s_{x x}(t, x)+\frac{C}{x} s_{x}(t, x)+s_{t}(t, x)=0
\end{aligned}
$$

Let us first solve (26) to extract $s$. It is equivalent to

$$
\left[s^{-1}(t, x)\right]_{x}=x^{-\alpha},
$$

hence

$$
s^{-1}(t, x)=\frac{x^{1-\alpha}}{1-\alpha}
$$

and

$$
s(t, x)=[x(1-\alpha)]^{\frac{1}{1-\alpha}} .
$$


With this scale function $s$, it follows from (27) that

$$
g(x)=\frac{\alpha}{2(\alpha-1)} \frac{1}{x}, \quad \text { that is, } C=\frac{\alpha}{2(\alpha-1)} .
$$

Applying Theorem 1, we obtain the following S.D.E.:

$$
R_{t}=z+B_{t}+\int_{0}^{t} \frac{\alpha}{2(\alpha-1)} \frac{1}{R_{s}} d s
$$

This is the equation of a Bessel process of dimension $d=1+\alpha /(\alpha-1)$, under the condition $d \geq 2 \Leftrightarrow \alpha \geq 1$. But with $\alpha \geq 1$, we have: $1 /(1-\alpha) \leq 0,1-\alpha \leq 0$ and $s(x)$ will be negative for positive values of $x$. Since a Bessel process can never be negative, let us choose

$$
R_{t} \stackrel{(d)}{\simeq}-\operatorname{Bes}_{z}^{1+\frac{\alpha}{\alpha-1}} \quad \text { with } \alpha>1
$$

that is, $\left(R_{t}\right)_{t \geq 0}$ is the opposite of a Bessel process starting from $z$ and of dimension $1+\alpha /(\alpha-1), \alpha>1$.

By Theorem 1, we get for the solution $\left(X^{1, \varepsilon}, Y^{1, \varepsilon}\right)$ of a 2-dimensional equation of type $\left(E_{1}\right)$ with any suitable functions $b$ and $\sigma$,

$$
\lim _{\varepsilon \rightarrow 0} Y_{T^{1, \varepsilon}}^{1, \varepsilon} \stackrel{(d)}{\simeq}\left\{\frac{1}{R_{1}(1-\alpha)}\right\}^{\frac{1}{\alpha-1}}
$$

The density of Bessel processes being known [cf. Revuz and Yor (1994), page 426], we can deduce the one of $\lim _{\varepsilon \rightarrow 0} Y_{T^{1, \varepsilon}}^{1, \varepsilon}$ :

$$
p(x)=\left(\frac{x}{z}\right)^{\frac{1}{2(\alpha-1)}} \exp \left\{-\frac{\left(x^{2}+z^{2}\right)}{2}\right\} \frac{x}{[x(\alpha-1)]^{\frac{1}{\alpha-1}}} I_{\frac{1}{2(\alpha-1)}}(x z),
$$

where $I_{\nu}$ denotes the Bessel function of index $\nu$.

5.4. Another Bessel process. Let us now consider the situation where $\delta \neq 0$. We impose here $\delta>-1$, that the functions $f$ and $g$ are of the same form as in 5.3 , that is, $f \equiv 1$ and $g(t, x)=C / x$; and we choose a function $\phi$ defined by

$$
\phi(t, x)=e^{2 x} .
$$

This function satisfies the required hypotheses for Theorem 1: it is indeed Lipschitz continuous and lower bounded on the set $\left\{x \in\left[0, \varepsilon^{2}\right]\right\}$. In this case, the system (4) becomes

$$
\begin{aligned}
& s^{\prime}{ }_{0} s^{-1}(t, x)=e^{-\frac{x}{2}}, \\
& \frac{1}{2} s_{x x}(t, x)+\frac{C}{x} s_{x}(t, x)+s_{t}(t, x)=\delta\left(s_{x}(t, x)\right)^{2}
\end{aligned}
$$


From (29) we deduce

$$
s(t, x)= \begin{cases}\ln x, & \text { if } x>0 \\ \ln |x|, & \text { if } x<0\end{cases}
$$

REMARK. This function $s$ is not bijective on $\mathbb{R}$ but it is on $\mathbb{R}_{+}$. This is sufficient since we need it to be bijective only on the life space of the process $\left(R_{t}\right)_{t \geq 0}$ (cf. proof of Theorem 1). As this one will turn out to be a Bessel process, it lives on $\mathbb{R}_{+}$and the function $s$ satisfies the hypotheses.

Taking $C=\delta+1 / 2,(30)$ is satisfied, so we choose

$$
g(t, x)=\left(\delta+\frac{1}{2}\right) \frac{1}{x} .
$$

We deduce then the limit law of $Y_{T^{1, \varepsilon}}^{1, \varepsilon}$ when $\varepsilon \rightarrow 0$ with the S.D.E. satisfied by $\left(R_{t}\right)_{t \geq 0}$ :

$$
R_{t}=z+B_{t}+\int_{0}^{t}\left(\delta+\frac{1}{2}\right) \frac{1}{R_{s}} d s .
$$

This is the equation of a Bessel process of dimension $2(\delta+1): R_{t} \sim \mathscr{B} e s_{z}^{2(\delta+1)}$ when $\delta>-1$. Then

$$
\lim _{\varepsilon \rightarrow 0} Y_{T^{1, \varepsilon}}^{1, \varepsilon} \stackrel{(d)}{\simeq} s\left(1, R_{1}\right) \stackrel{(d)}{\simeq} \ln \left(R_{1}\right)
$$

From the known density of Bessel processes, it follows that the one of the required limit law $\lim _{\varepsilon \rightarrow 0} Y_{T^{1, \varepsilon}}^{1, \varepsilon}$ is given by

$$
p(x)=\frac{x^{\delta+1} \ln x}{z^{\delta}} \exp \left\{-\left(\frac{x^{2}+z^{2}}{2}\right)\right\} I_{\delta}(x z),
$$

where $I_{\nu}$ denotes the Bessel function of index $\nu$.

5.5. Process associated to the porous media equation. Let us now recall the stochastic interpretation of the porous media equation

$$
u_{t}=\frac{1}{2}\left(u^{2 m+1}\right)_{x x}
$$

studied in Benachour, Chassaing, Roynette and Vallois (1996). The authors associate to it the following S.D.E.:

$$
\left\{\begin{array}{l}
d R_{t}=u^{m}\left(t, R_{t}\right) d B_{t}, R_{0}=0, \\
u(t, .) \text { is the density of } R_{t}, t>0 \\
m>0
\end{array}\right.
$$

and prove that this equation admits a unique solution $\left(R_{t}\right)_{t \geq 0}$. Its density $u$, solution of the porous media equation, is the Barenblatt-Pattle density

$$
u(t, x)=\frac{1}{t^{\beta}} \chi\left(\frac{x}{t^{\beta}}\right)=\frac{1}{t^{\beta}}\left\{a_{m}^{2}-\frac{1}{(2 m+1)(m+1)} \frac{x^{2}}{t^{2 \beta}}\right\}_{+}^{\frac{1}{2 m}},
$$


with

$$
\begin{aligned}
\beta & =\frac{1}{2 m+2}, \\
a_{m} & =\left\{\frac{m^{\frac{1}{2}}}{[(2 m+1)(m+1)]^{\frac{1}{2}} B\left(\frac{2 m+1}{2 m}, \frac{1}{2}\right)}\right\}^{\frac{m}{m+1}}, \\
\gamma_{m} & =a_{m}\left(\frac{(2 m+1)(m+1)}{m}\right)^{\frac{1}{2}}, \\
\chi(x) & =a_{m}{ }^{\frac{1}{m}}\left\{1-\left(\frac{x}{\gamma_{m}}\right)^{2}\right\}_{+}^{\frac{1}{2 m}} .
\end{aligned}
$$

Let us now return to our problem. We impose again $\delta=0$ and we consider the case of a function $\phi$ defined by

$$
\phi(t, x)=\frac{1}{u^{2 m}(t, x)},
$$

where $u$ is the Barenblatt-Pattle density given by (32).

Our aim is to use $\phi$ to define a S.D.E. as follows:

$$
\left\{\begin{array}{l}
X_{t}=\int_{0}^{t} \phi\left(X_{s}, Y_{s}\right) d s \\
Y_{t}=B_{t}
\end{array}\right.
$$

In other words, we need $\phi\left(., B_{t}\right)$ to be well defined, that is $u\left(., B_{t}\right) \neq 0$. Looking at the explicit formula of $u$, we remark that

$$
u(t, x)>0 \Longleftrightarrow|x|<\gamma_{m} t^{\frac{1}{2 m+2}} .
$$

On the other hand, using the law of the iterated logarithm, we obtain that for small $t$,

$$
\left|B_{t}\right| \leq c \cdot t^{\alpha} \text { a.s. } \quad \text { with } \alpha>\frac{1}{2} \text { and a constant } c .
$$

Since $m>0$, we can always find an $\alpha>1 / 2$ such that $\alpha>1 /(2 m+2)$, then we obtain

$$
\left|B_{t}\right| \leq c t^{\frac{1}{2 m+2}} \text { a.s. }(t \text { small })
$$

so we can take $B_{t} \in\left[-\gamma_{m} t^{\frac{1}{2 m+2}} ; \gamma_{m} t^{\frac{1}{2 m+2}}\right]$ and in this interval, the defined function $\phi$ has the required properties, that is $\phi\left(., B_{t}\right)$ is well-defined.

We can then apply our result: since $\delta=0$, we have to solve the system (22) in order to find some functions $f, g$ and $s$. Here (22) becomes

$$
\left\{\begin{array}{l}
{\left[\left(s_{x} \cdot f\right)_{o} s^{-1}\right](t, x)=u^{m}(t, x)} \\
\frac{1}{2} f^{2}(t, x) s_{x x}(t, x)+g(t, x) s_{x}(t, x)+s_{t}(t, x)=0
\end{array}\right.
$$


If we choose $g \equiv 0$ and

$$
f(t, x)=u^{m}(t, x),
$$

the process $\left(R_{t}\right)_{t \geq 0}$ solution of (4) is the process associated to the porous media equation. The preceeding system is then solved with the natural scale function $s(t, x)=x$ for the operator

$$
L=\frac{1}{2} u^{2 m} \frac{\partial^{2}}{\partial x^{2}}+\frac{\partial}{\partial t} .
$$

Then, for any S.D.E. of the form $\left(E_{1}\right)$ with suitable functions $\sigma$ and $b$, we get by Theorem 1

$$
\lim _{\varepsilon \rightarrow 0} Y_{T^{1, \varepsilon}}^{1, \varepsilon} \stackrel{(d)}{\simeq} Y_{T} \stackrel{(d)}{\simeq} s\left(1, R_{1}\right) \stackrel{(d)}{\simeq} R_{1}
$$

Hence the density of the limit law is given by

$$
p(x)=\left\{a_{m}^{2}-\frac{1}{(2 m+1)(m+1)} x^{2}\right\}_{+}^{\frac{1}{2 m}} .
$$

5.6. S.D.E. with multiplicative noise. We stay finally in the situation where $\delta=0$ and we consider the following function:

$$
\phi(x)=\left\{\frac{1}{\left(b-\frac{2 a}{b}\right) x}\right\}^{2}
$$

with $a$ and $b$ some constants such that $b^{2} \neq 2 a$.

With the arguments we have already used in the examples 5.2 and 5.3, we are allowed to apply our result to such a function. The system (22) that we have then to solve becomes

$$
\begin{aligned}
& {\left[\left(s_{x} . f\right)_{o} s^{-1}\right](t, x)=\left(b-\frac{2 a}{b}\right) x,} \\
& \frac{1}{2} f^{2}(t, x) s_{x x}(t, x)+g(t, x) s_{x}(t, x)+s_{t}(t, x)=0 .
\end{aligned}
$$

Let us suppose that all the functions to determine are independent of their first variable, and let us choose simple multiplicative functions:

$$
f(x)=b x \text { and } g(x)=a x .
$$

Then the system (33)-(34) becomes

$$
\begin{aligned}
& s_{x}(x) \cdot b x=\left(b-\frac{2 a}{b}\right) s(x), \\
& \frac{1}{2} b^{2} x^{2} s_{x x}(x)+a x s_{x}(x)=0 .
\end{aligned}
$$


Let us solve (36) in order to get the explicit formula for $s$

$$
\left[\ln s_{x}(x)\right]^{\prime}= \begin{cases}-\frac{2 a}{b^{2}}[\ln x]^{\prime}, & \text { if } x>0, \\ -\frac{2 a}{b^{2}}[\ln |x|]^{\prime}, & \text { if } x<0\end{cases}
$$

then

$$
s_{x}(x)= \begin{cases}x^{-\frac{2 a}{b^{2}}}, & \text { if } x>0 \\ |x|^{-\frac{2 a}{b^{2}}}, & \text { if } x<0\end{cases}
$$

and we deduce

$$
s(x)=\frac{b^{2}}{b^{2}-2 a}|x|^{1-\frac{2 a}{b^{2}}}
$$

REMARK. We can make here the same remark as in the example 5.4 about the bijectivity of $s$ since we will see that it is sufficient to fix a positive starting point for the process $\left(R_{t}\right)_{t \geq 0}$ solution of (4) to make it live in $\mathbb{R}_{+}$.

Applying Theorem 1 leads us to consider the following S.D.E.:

$$
R_{t}=z+a \int_{0}^{t} R_{s} d s+b \int_{0}^{t} R_{s} d B_{s}
$$

with a fixed $z>0$.

The solution of this equation is known and has the following explicit expression

$$
R_{t}=z \cdot \exp \left\{\left(a-\frac{b^{2}}{2}\right) t+b B_{t}\right\} .
$$

It follows that, for any suitable functions $\sigma$ and $b$, the solution $\left(X^{1, \varepsilon}, Y^{1, \varepsilon}\right)$ of an equation of the form $\left(E_{1}\right)$ satisfies

$$
\lim _{\varepsilon \rightarrow 0} Y_{T^{1, \varepsilon}}^{1, \varepsilon} \stackrel{(d)}{\simeq} Y_{T} \stackrel{(d)}{\simeq} s\left(1, R_{1}\right) \stackrel{(d)}{\simeq} \frac{b^{2}}{b^{2}-2 a}\left(R_{1}\right)^{1-\frac{2 a}{b^{2}}}
$$

And we deduce from (37) the density of the limit law

$$
p(x)=z^{1-\frac{2 a}{b^{2}}} \frac{1}{\sqrt{2 \pi}} \frac{b^{2}}{b^{2}-2 a} \exp \left\{\left(a-\frac{b^{2}}{2}\right)\left(1-\frac{2 a}{b^{2}}\right)\right\} \exp \left\{\left(b-\frac{2 a}{b}\right) x-\frac{x^{2}}{2}\right\}
$$

\section{REFERENCES}

Benachour S., Chassaing P., Roynette B. and Vallois P. (1996). Processus associés à l'équation des milieux poreux. In Annali della scuola normale superiore di Pisa. Scienze Fisiche e Matematiche.

Chambert-Loir A. and Fermigier S. (1996). Exercices de mathématiques pour l'agrégation. Analyse 3. Editions Masson. 
FAURE O. (1992). Simulation du mouvement brownien et des diffusions. Thèse de doctorat, Ecole Nationale des Ponts et Chaussées.

Revuz D. and Yor M. (1994). Continuous Martingales and Brownian Motion. Springer, Berlin.

INSTITUT ELIE CARTAN

UNIVERSITÉ DE NANCY I

B.P. 239

54506 VANDOEUVRE-Les-NANCY CEDEX

FRANCE

E-MAIL: bernard.roynette@iecn.u-nancy.fr sophie.wantz@iecn.u-nancy.fr 